\title{
Анализ влияния неодномерных эффектов на отпирающий ток управления тиристорных структур на основе $4 \mathrm{H}-\mathrm{SiC}$
}

\author{
(C) С.Н. Юрков ${ }^{1}$, T.T. Мнацаканов ${ }^{1}$, М.Е. Левинштейн ${ }^{2, \uparrow}$, А.Г. Тандоев ${ }^{1}$, J.W. Palmour ${ }^{3}$ \\ ${ }^{1}$ Всероссийский электротехнический институт им. В.И. Ленина, \\ 111250 Москва, Россия \\ ${ }^{2}$ Физико-технический институт им. А.Ф. Иоффре Российской академии наук, \\ 194021 Санкт-Петербург, Россия \\ ${ }^{3}$ Wolfspeed, A Cree company, 3026 East Cornwallis Rd., \\ Research Triangle Park NC 27709, USA \\ ฯ E-mail: melev@nimis.ioffe.rssi.ru
}

(Получена 24 мая 2016 г. Принята к печати 14 июня 2016 г.)

Рассмотрено влияние неодномерных эффектов, обусловленных растеканием тока управления в базовом слое, на величину отпирающего тока управления тиристоров на основе $4 H$-SiC. Показано, что реализующийся в $4 H$-SiC тиристорах новый механизм переключения приводит к зависимости отпирающего тока управления от параметров тиристора, качественно отличающейся от соответствующей зависимости в традиционных кремниевых тиристорах.

DOI: 10.21883/FTP.2017.02.44111.8335

\section{1. Введение}

Высоковольтные тиристоры находят широкое применение в мощных преобразовательных схемах передающих линий, компенсаторах реактивной мощности, мощных импульсных генераторах, оборудовании автоматического контроля, в схемах управления эксимерных лазеров и цепях синхронизации мощных лазерных систем.

Высоковольтные тиристоры на основе карбида кремния во многих отношениях демонстрируют важные преимущества перед тиристорами на основе $\mathrm{Si}$ и GaAs, в особенности в высокотемпературных применениях, требующих большого блокирующего напряжения единичного элемента и высокой плотности тока [1-4].

В работах [5-7] было обращено внимание на то, что включение $\mathrm{SiC}$-тиристоров происходит при весьма больших токах, протекающих через структуру. При этом включение осуществляется не при низком уровне инжекции в блокирующей базе тиристора, как в хорошо исследованных кремниевых тиристорах, а при промежуточном между низким и высоким уровнями инжекции.

Этот эффект обусловлен несколькими особенностями $\mathrm{SiC}$-тиристоров. Во-первых, в сильно легированном $p^{++}$-эмиттере высоковольтного $\mathrm{SiC}$-тиристора акцепторный уровень ионизован не полностью в связи с достаточно большой энергией ионизации даже наиболее мелкого акцептора (Al) в $\mathrm{SiC}$. При энергии ионизации $\mathrm{Al}$ в $4 H-\mathrm{SiC}$, равной $0.19-0.24$ эВ [8], и уровне легирования $N_{a} \sim 2 \cdot 10^{19} \mathrm{~cm}^{-3}$ только $(1-2) \%$ атомов $\mathrm{Al}$ оказываются ионизованными при комнатной температуре. В результате коэффициент инжекции $p^{++}-n$-перехода между сильно легированным $p^{++}$-эмиттером и тонкой $n$-базой оказывается весьма низким. Во-вторых, отношение ширины блокирующей базы к диффузионной длине неосновных носителей в $\mathrm{SiC}$-тиристорах, как правило, существенно больше, чем в кремниевых структурах. Наконец, на границе между $p^{++}$-эмиттером и тонкой $n$-базой нередко существует тонкий слой с временем жизни неосновных носителей, значительно меньшим, чем в основной части тонкой $n$-базы. Наличие такого слоя также снижает коэффициент инжекции $p^{++}-n$-перехода [5].

Включение тиристора при промежуточном уровне инжекции в блокирующей базе приводит не только к количественным изменениям параметров включения, но также и к качественному изменению происходящих при этом процессов. В работах [5,9] были проанализированы особенности поведения критического заряда включения, а в работах $[10,11]$ - особенности процесса включения в условиях этого нового механизма переключения. В работе [11] анализ отпирающего тока управления был проделан в рамках одномерного приближения. В реальных тиристорных структурах, однако, процесс включения током управления принципиально является неодномерным.

Цель данной работы - исследование влияния нового механизма включения на характеристики тиристора в неодномерном приближении.

\section{2. Постановка задачи}

Хорошо известно, что условие нахождения тиристора в выключенном состоянии имеет вид:

$$
K=\alpha_{1}+\alpha_{2}=\gamma_{1} \alpha_{T 1}+\gamma_{2} \alpha_{T 2}<1
$$

где $\alpha_{1}=\gamma_{1} \alpha_{T 1}$ и $\alpha_{2}=\gamma_{2} \alpha_{T 2}-$ коэффициенты усиления составных транзисторов тиристорной структуры в схеме с общей базой. Индексы „1“ относятся к транзистору с узкой базой, индексы „2“ (блокирующей) базой соответственно. Параметры $\gamma_{1}$ и $\gamma_{2}-$ коэффициенты инжекции переходов $p^{++}-n$ - 
и $n^{+}-p$-переходов эмиттер-база (для $\mathrm{SiC}$-тиристоров характерно наличие тонкой $n$-базы и блокирующей базы $p$-типа), $\alpha_{T 1}$ и $\alpha_{T 2}$ - коэффициенты переноса тонкой и блокирующей баз соответственно.

При выполнении противоположного условия $K>1$ тиристор оказывается во включенном состоянии. Таким образом, очевидно, что для того чтобы тиристор включился, необходимо, чтобы с ростом тока управления возрастала по крайне мере одна из четырех величин, входящих в выражение (1).

В традиционных тиристорах на основе $\mathrm{Si}$ такой величиной является коэффициент инжекции эмиттерного перехода $\gamma_{1}$. Физической причиной, обусловливающей реализацию такого $\gamma$-механизма включения тиристора, является специально вводимая в конструкцию прибора технологическая шунтировка. Действительно, при небольшом напряжении, приложенном к шунтированному переходу, сопротивление даже прямо смещенного $p-n$-перехода очень велико. Практически весь ток протекает через омическое сопротивление шунтировки, инжекция неосновных носителей в тонкую базу отсутствует, и значение $\gamma_{1}$ близко к нулю. Однако сопротивление прямо смещенного перехода экспоненциально падает с ростом прямого напряжения на переходе, и при достаточно большом значении управляющего сигнала ток в основном начинает протекать через прямо смещенный $p-n$-переход. При этом коэффициент инжекции резко возрастает, стремясь к максимальному значению $\gamma_{10}$, определяемому параметрами эмиттера и базы $[12,13]$. В дальнейшем мы будем называть такой механизм включения $\gamma$-механизмом.

В тиристорных структурах на основе $\mathrm{SiC}$ технологическая шунтировка отсутствует. Поэтому в первом приближении можно считать, что коэффициенты инжекции обоих переходов эмиттер-база не зависят от тока. Как показано в работах [5-7], параметром, возрастание которого приводит к включению тиристора, является коэффициент переноса блокирующей базы $\alpha_{T 2}$. Переход от низкого уровня инжекции к высокому в толстой блокирующей базе тиристора сопровождается ростом параметра $\alpha_{T 2}$ от значения $\left(\alpha_{T 2}\right)_{L}$ до значения $\left(\alpha_{T 2}\right)_{H}$ :

$$
\begin{gathered}
\left(\alpha_{T 2}\right)_{L}=1 / \operatorname{ch}\left(W_{p} / L_{n}\right), \\
\left(\alpha_{T 2}\right)_{H}=\frac{b}{b+1}+\frac{1}{b+1} \frac{1}{\operatorname{ch}\left(W_{p} / L_{a}\right)},
\end{gathered}
$$

где $W_{p}-$ ширина блокирующей базы $p$-типа, $L_{n}=\sqrt{D_{n}\left(\tau_{n}\right)_{L}}-$ диффузионная длина электронов в блокирующей базе, $L_{a}=\sqrt{\frac{2 b}{b+1} D_{p}\left(\tau_{n}\right)_{H}}-$ амбиполярная диффузионная длина, $b=\mu_{n} / \mu_{p}$ - отношение подвижности электронов к подвижности дырок, $D_{n}$ и $D_{p}-$ коэффициенты диффузии электронов и дырок соответственно, $\left(\tau_{n}\right)_{L}$ и $\left(\tau_{n}\right)_{H}$ - времена жизни в блокирующей базе $p$-типа при низком и высоком уровнях инжекции.

Численные расчеты показывают, что для высоковольтных $\mathrm{SiC}$-тиристоров разница между значениями $\left(\alpha_{T 2}\right)_{L}$ и $\left(\alpha_{T 2}\right)_{H}$ может быть чрезвычайно существенной. Так, для
$\mathrm{SiC}$-тиристорной структуры с блокирующим напряжением 12 кВ величины $\left(\alpha_{T 2}\right)_{L}$ и $\left(\alpha_{T 2}\right)_{H}$ составляли $(0.4-0.5)$ и (0.86-0.95) соответственно [13].

Режим, в котором зависимость $\alpha_{T 2}$ от тока является основным фактором, определяющим условие включения тиристора, предложено называть $\alpha$-механизмом [11].

Зависимость $\alpha_{T 2}(J)$, определяющая переход с ростом тока от значения $\left(\alpha_{T 2}\right)_{L}$ к значению $\left(\alpha_{T 2}\right)_{H}$ (выражение (2)), может быть определена только численно. В работах $[14,15]$ была предложена простая аппроксимация зависимости $\alpha_{T 2}(J)$, обеспечивающая приемлемую для практических расчетов точность в широком диапазоне значений плотности тока:

$$
\alpha_{T 2}(J)=\frac{\left(\alpha_{T 2}\right)_{L}+\left(\alpha_{T 2}\right)_{H} J / J_{0}}{1+J / J_{0}},
$$

где $J_{0}$ - параметр аппроксимации.

В работе [11] были получены условия реализации $\gamma$ - и $\alpha$-механизмов включения. Условием реализации $\gamma$-механизма является наличие утечки в эмиттерном переходе и выполнение неравенства

$$
\gamma_{10} \alpha_{T 1}+\gamma_{2}\left(\alpha_{T 2}\right)_{L}>1,
$$

где $\gamma_{10}$ - максимальное значение коэффициента инжекции эмиттерного перехода (без учета шунтировки). Условием реализации $\alpha$-механизма является система неравенств

$$
\begin{aligned}
& \gamma_{10} \alpha_{T 1}+\gamma_{2}\left(\alpha_{T 2}\right)_{H}>1, \\
& \gamma_{10} \alpha_{T 1}+\gamma_{2}\left(\alpha_{T 2}\right)_{L}<1 .
\end{aligned}
$$

Как отмечалось выше, в реальных тиристорных структурах процесс включения током управления принципиально является неодномерным. Из-за конечного сопротивления базовых слоев включение тиристора всегда

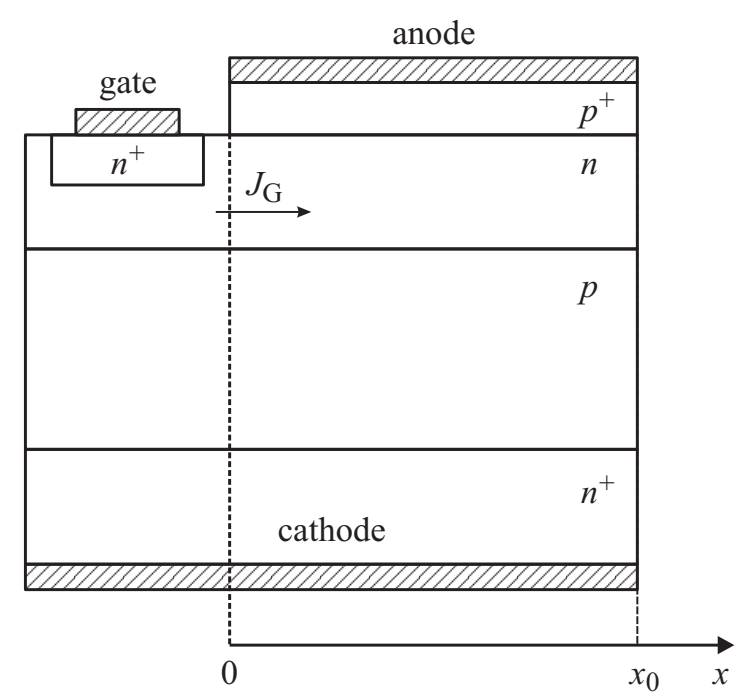

Рис. 1. Схема протекания тока управления для тиристорной структуры с легированием слоев, характерным для $\mathrm{SiC}$-тиристоров: блокирующая база $p$-типа, тонкая база $n$-типа, верхний эмиттер $p^{+}$-типа. Тиристор имеет вид прямоугольной полосы шириной $2 x_{0}$. 
происходит локально в областях, примыкающих к управляющему электроду. Рассмотрим влияние неодномерных эффектов, связанных с растеканием тока управления в базовых слоях, на примере структуры, представленной на рис. 1.

\section{3. Результаты и обсуждение}

Будем рассматривать тонкую базу тиристорной структуры как плоскую проводящую среду с листовым сопротивлением $R_{s}$. Поскольку листовое сопротивление толстой базы, как правило, на несколько порядков больше, чем тонкой, пренебрежем растеканием тока в широкой базе, Тогда уравнение, описывающее распределение напряжения на $p^{+}-n$-эмиттерном переходе вдоль оси $x$, имеет вид [16] (см. также $[17,18])$ :

$$
\begin{aligned}
\frac{d^{2} U_{e}}{d x^{2}} & =-R_{s} J_{R G}(x) \\
& =-R_{S} \frac{J(x)}{\alpha_{1}}\left[\alpha_{1}-1+\gamma_{2} \frac{\left(\alpha_{T 2}\right)_{L}+\left(\alpha_{T 2}\right)_{H} J / J_{0}}{1+J / J_{0}}\right] .
\end{aligned}
$$

Уравнение (7) описывает распределение напряжения на эмиттерном переходе. В точке $x=0$ выполняется равенство:

$$
\left.\frac{d U_{e}}{d x}\right|_{x=0}=-R_{s} J_{G} .
$$

В точке $x=x_{0}$ вследствие симметрии выбранной структуры справедливо равенство

$$
\left.\frac{d U_{e}}{d x}\right|_{x=x_{0}}=0 .
$$

Здесь $U_{e}-$ напряжение на $p^{+}-n$-эмиттерном переходе, $J_{G}$ - линейная плотность тока управления в точке $x=0$. Граничное условие (8) соответствует поступлению в базовый слой в точке $x=0$ внешнего тока управления $J_{G}$. Величина $J_{R G}$ в каждой точке $x$ характеризует потерю тока управления на поддержание функционирования внутренней регенеративной обратной связи тиристора.

Плотность тока, протекающего через тиристорную структуру, $J(x)$, связана с напряжением $U_{e}(x)$ следующим соотношением [16]:

$$
J=\frac{J_{s} \alpha_{T 1} \exp \left(q U_{e} / k T\right)}{\left[1-\gamma_{2} \alpha_{T 2}\right]},
$$

где $J_{s}-$ дырочная составляющая тока насыщения эмиттерного $p^{+}-n$-перехода.

Нелинейное уравнение (7) не может быть решено в аналитическом виде. Численное решение (7) представляет довольно сложную задачу, поскольку уравнение имеет два решения, относящихся к двум возможным достаточно близко расположенным статическим состояниям тиристора, соответствующим положительной и отрицательной ветвям статической вольт-амперной характеристики. (Третья (положительная) ветвь статической вольт-амперной характеристики, соответствующая полностью включенному тиристору, отстоит по току достаточно далеко от первых двух ветвей, и ее наличие не вызывает вычислительных трудностей).

Для численного решения системы (7)-(9) был использован следующий искусственный прием. Введем в рассмотрение емкость эмиттерного перехода $C$, которая будет заряжаться током управления, втекающим в базу. Тогда при начальном нулевом напряжении на эмиттерном переходе в результате протекания тока управления емкость зарядится до напряжения, соответствующего равновесному состоянию тиристора в выключенном состоянии. Таким образом, статическая задача заменяется динамической, что позволяет выделить одно из искомых решений. Вместо системы (7)-(9) будем решать следующую динамическую задачу:

$$
\begin{gathered}
\frac{\partial U_{e}}{\partial t}=\frac{1}{R_{s} C} \frac{\partial^{2} U_{e}}{\partial x^{2}} \\
+\frac{J}{\alpha_{1} C}\left[\alpha_{1}-1+\gamma_{2} \frac{\left(\alpha_{T 2}\right)_{L}+\left(\alpha_{T 2}\right)_{H} J / J_{0}}{1+J / J_{0}}\right], \\
\left.\frac{\partial U_{e}}{\partial x}\right|_{x=0}=-R_{s} J_{G}, \\
\left.\frac{\partial U_{e}}{\partial x}\right|_{x=x_{0}}=0, \\
U_{e}(t=0)=0 .
\end{gathered}
$$

Величина $C$, являющаяся параметром численной модели, определяет скорость сходимости вычислительного процесса и не влияет на искомое решение.

Расчет отпирающего тока управления $J_{G T}$ осуществлялся следующим образом. Для каждого значения $J_{G}$ решалась численно задача $(11)-(14)$. При этом величина $J_{G}$ увеличивалась до тех пор, пока не начинался неограниченный рост тока $J(t)$, соответствующий включению тиристора. При расчетах использовались параметры полупроводниковой структуры, соответствующие $4 H$-SiC тиристору 18 кВ класса, приведенные в работе [10]: $\gamma_{1}=0.146, \alpha_{T 1}=0.938, \gamma_{2}=1,\left(\alpha_{T 2}\right)_{L}=0.368$, $\left(\alpha_{T 2}\right)_{H}=0.8795, J_{0}=0.06 \mathrm{~A} / \mathrm{cm}^{2}, J_{s}=7.3 \cdot 10^{-48} \mathrm{~A} / \mathrm{cm}^{2}$.

На рис. 2 приведена зависимость отпирающего тока управления $J_{G T}$ от полуширины эмиттерной полосы $x_{0}$ при двух значениях листового сопротивления узкой базы $R_{s}$ (кривые 1 и 2). При малых $x_{0}$ величина $J_{G T}$ растет пропорционально $x_{0}$ и не зависит от сопротивления базы, что соответствует ситуации, близкой к одномерному случаю [11]. С увеличением $x_{0}$ рост $J_{G T}$ замедляется, и отпирающий ток управления стремится к некоторой предельной величине, зависящей от $R_{s}$.

Как видно из кривых 1 и 2, практически полное насыщение зависимости $J_{G T}\left(x_{0}\right)$ при реалистических значениях $R_{s}$ в случае „чистого“ $\alpha$-механизма включения соответствует значениям $x_{0} \sim 400-600$ мкм, что 


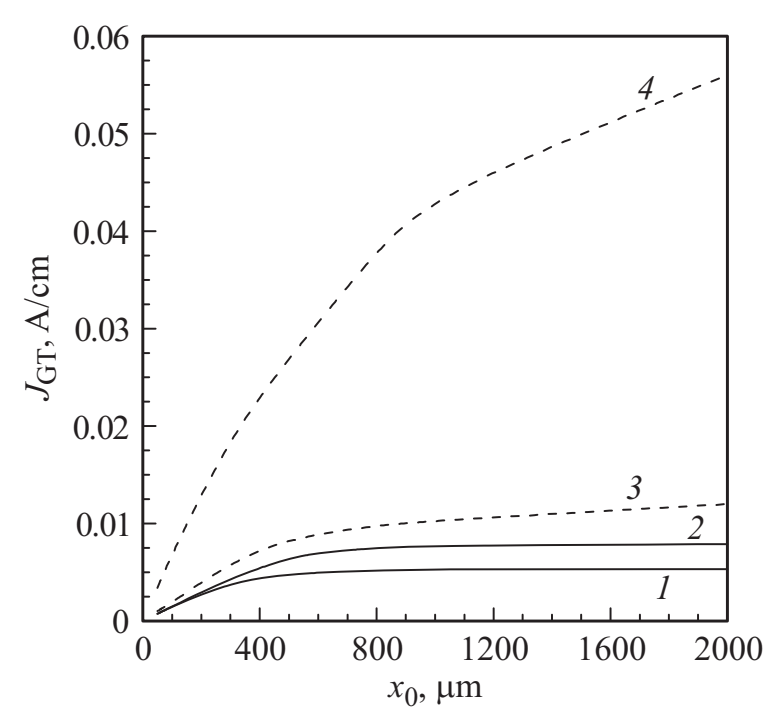

Рис. 2. Зависимость плотности отпирающего тока управления $J_{G T}$ тиристора с $\alpha$-механизмом включения от полуширины эмиттерной полосы $x_{0}$. Сплошные кривые соответствуют „чистому“ $\alpha$-механизму включения: предполагается отсутствие утечки в $p^{+}$-эмиттере. $R_{s}($ Ом): $1-1000,2-453$. Пунктирные кривые соответствуют $\alpha$-механизму включения с учетом равномерно распределенной утечки с эффективным сопротивлением $R_{d}$ в $p^{+}-n$-эмиттере: $3-R_{s}=453$ Ом, $R_{d}=50 \mathrm{OM} \cdot \mathrm{cm}^{2}, 4-R_{s}=453 \mathrm{OM}, R_{d}=5 \mathrm{OM} \cdot \mathrm{cm}^{2}$.

значительно меньше, чем размер современных мощных $\mathrm{SiC}$-тиристоров $[10,11,16,19]$. Физическая причина такой зависимости $J_{G T}\left(x_{0}\right)$ заключается в следующем. Протекание тока управления приводит к падению напряжения на сопротивлении базы. В свою очередь это приводит к снижению напряжения на эмиттерном переходе [20]. В результате напряжение $U_{e}$ максимально при $x=0$ и снижается при удалении от края тиристорной структуры. Из выражений (8) и (10) следует, что ток регенерации $J_{R G}$ экспоненциально зависит от $U_{e}$; поэтому он быстро спадает при удалении от края структуры. Поскольку часть тока управления, протекающая в базе в поперечном оси $x$ направлении, идет на компенсацию $J_{R G}$, ток управления в $n$-базе локализован в узкой области вблизи точки $x=0$, и величина отпирающего тока управления $J_{G T}$ при больших значениях $x_{0}$ перестает зависеть от размера эмиттерной полосы.

Как отмечалось в работе [14], из анализа экспериментальных зависимостей тока удержания $4 \mathrm{H}$ - $\mathrm{SiC}$ тиристоpa 12-кВ класса следует наличие в тиристоре эффективного „непреднамеренного“ шунтирования перехода $p^{+}$-эмиттер-тонкая $n$-база. Уровень такого шунтирования характеризуется величиной поверхностного сопротивления $R_{d}$. Хорошо известно, что шунтировка может быть обусловлена не только прямым введением шунтов в эмиттер, но и, например, наличием дефектов в поверхностном слое эмиттера. Как показано в работе [11], в карбид-кремниевых тиристорах имеются несколько механизмов, ответственных за появление избыточных токов утечки перехода $p^{+}$-эмиттер- $n$-база. Во-первых, очень высокий уровень легирования $p^{+}$-эмиттера обусловливает возникновение структурных дефектов, обусловливающих очень малые значения времени жизни неосновных носителей в эмиттере [21]. Во-вторых, высокая температура роста сильно легированного $p^{+}$-слоя приводит, вследствие механических напряжений и взаимодиффузии, к появлению в $n$-слое вблизи металлургической границы перехода нарушенного слоя с очень малыми временами жизни [9].

С учетом поверхностного сопротивления $R_{d}$ уравнение (11) будет иметь вид

$$
\begin{aligned}
& \frac{\partial U_{e}}{\partial t}=\frac{1}{R_{s} C} \frac{\partial^{2} U_{e}}{\partial x^{2}} \\
& +\frac{J}{\alpha_{1} C}\left[\alpha_{1}-1+\gamma_{2} \frac{\left(\alpha_{T 2}\right)_{L}+\left(\alpha_{T 2}\right)_{H} J / J_{0}}{1+J / J_{0}}\right]+\frac{U_{e}}{R_{d} C} .
\end{aligned}
$$

Рассчитанные с учетом наличия распределенного поверхностного сопротивления зависимости $J_{G T}\left(x_{0}\right)$ представлены кривыми 3 и 4 на рис. 2 для двух характерных значений $R_{d}[11]$. Видно, что введение распределенного поверхностного сопротивления, как и следовало ожидать, приводит к росту необходимого для включения тока управления. Изменяется также характер зависимости $J_{G T}\left(x_{0}\right): J_{G T}$ монотонно возрастает с ростом $x_{0}$, теперь ток управления расходуется не только на поддержание механизма регенеративной обратной связи, но и на восполнение потерь, связанных с утечкой в эмиттерном переходе. При этом если ток регенерации $J_{R G}$ зависит от $U_{e}$ экспоненциально и поэтому быстро спадает при удалении от края эмиттера, то ток утечки зависит от $U_{e}$ линейно. Таким образом, введение распределенной поверхностной утечки позволяет реализовать ситуацию, когда насыщение зависимости $J_{G T}\left(x_{0}\right)$ отсутствует, и сигнал тока управления проникает на значительное расстояние от электрода управления в глубь тонкой базы.

Интересно сравнить зависимости $J_{G T}\left(x_{0}\right)$ для тиристорных структур с $\alpha$ - и $\gamma$-механизмами включения. Как отмечалось выше, для реализации $\gamma$-механизма включения необходимо обеспечить рост величины коэффициента инжекции эмиттера, примыкающего к узкой базе $\gamma_{1}$ с ростом тока. Практически этого легче всего добиться, введя в конструкцию тиристорной структуры периферическую шунтировку, как показано на рис. 3.

Заметим, что в данном случае величина $x_{0}$ характеризует полную ширину эмиттерной полосы.

В отличие от тиристора с $\alpha$-механизмом включения, для расчета величины отпирающего тока управления тиристора с $\gamma$-механизмом может быть получено простое аналитическое выражение. Можно показать, что для $\gamma$-тиристоров в статическом состоянии, предшествующем включению, ток регенерации пренебрежимо мал по сравнению с током утечки, т.е. величина тока управления идет в основном на компенсацию потерь, связанных с утечкой в эмиттерном переходе. 


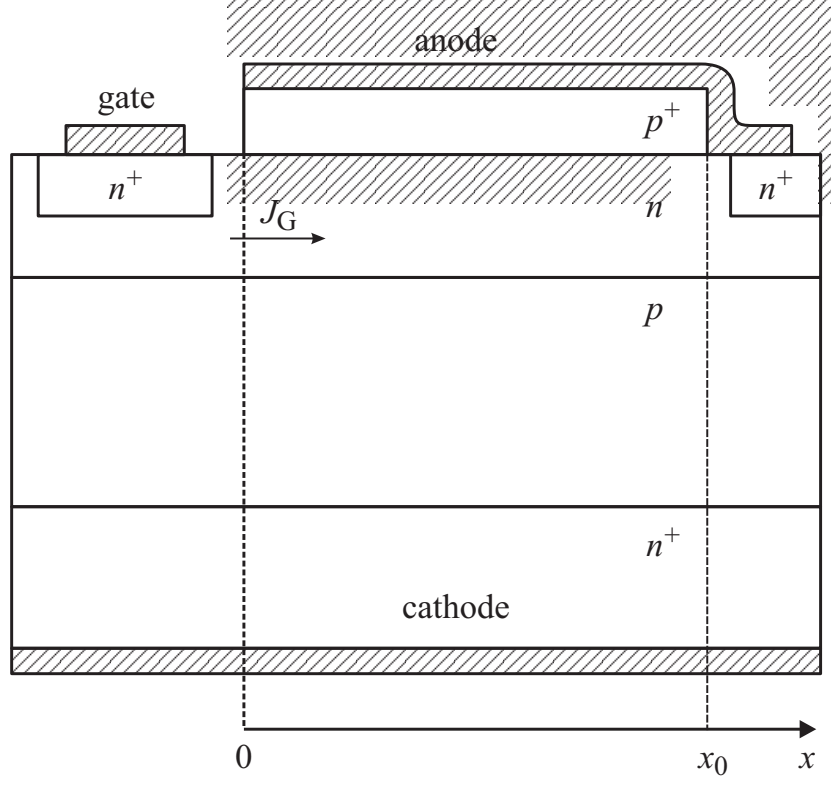

Рис. 3. Схема протекания тока управления для тиристорной структуры с легированием слоев, характерным для $\mathrm{SiC}$-тиристоров: блокирующая база $p$-типа, тонкая база $n$-типа, верхний эмиттер $p^{+}$-типа. Тиристор имеет вид прямоугольной полосы шириной $x_{0}$. Периферийный шунт располагается со стороны, противоположной электроду управления.

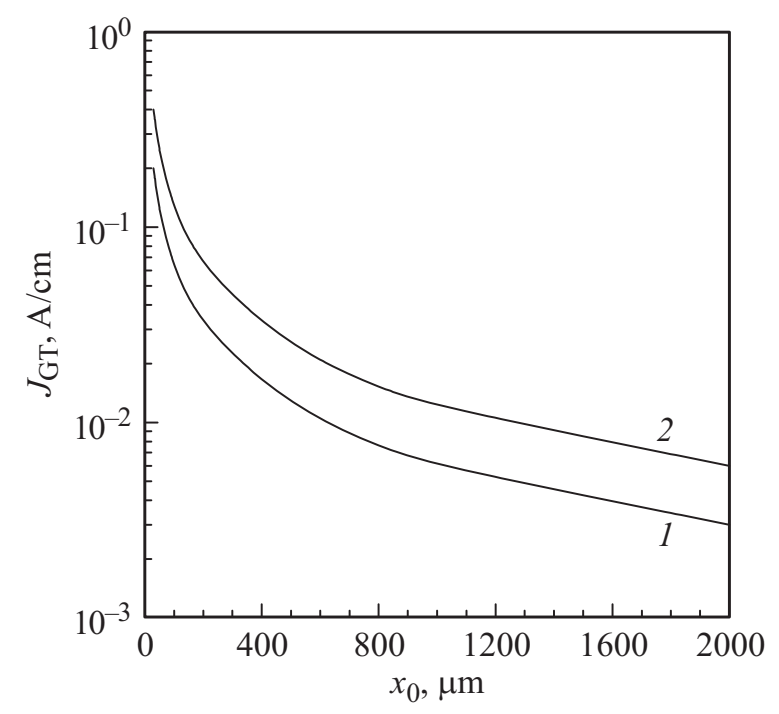

Рис. 4. Зависимость отпирающего тока управления $\gamma$-тиристора с периферийным шунтом от ширины эмиттерной полосы $x_{0} . R_{s}(\mathrm{OM}): 1-1000,2-453$.

В этом случае уравнение, описывающее распределение напряжения на $p^{+}-n$-переходе вдоль эмиттерной полосы имеет вид:

$$
\begin{gathered}
\frac{d^{2} U_{e}}{d x^{2}}=0, \\
\left.\frac{d U_{e}}{d x}\right|_{x=0}=-R_{s} J_{G}, \\
U_{e}\left(x=x_{0}\right)=0 .
\end{gathered}
$$

Решение системы (15)-(17) имеет вид

$$
U_{e}(x)=U_{e \max }\left(1-\frac{x}{x_{0}}\right),
$$

где

$$
U_{e \max }=R_{s} J_{G} x_{0} .
$$

Примем, что в состоянии, непосредственно предшествующем включению, $U_{e \max }=U_{e 0}$, где для $4 H-\mathrm{SiC}$ тиристоров с приемлемой для практических расчетов точностью можно положить $U_{e 0} \approx 2.85 \mathrm{~B}[22,23]$. Тогда

$$
J_{G T}=\frac{U_{e 0}}{R_{s} x_{0}}
$$

Таким образом, для $\gamma_{1}$-тиристора (в том числе для обычного тиристора на основе кремния) зависимость отпирающего тока управления от ширины эмиттерной полосы носит гиперболический характер (рис. 4).

При малых $x_{0}$ величина $J_{G T}$ очень велика и спадает при увеличении $x_{0}$, стремясь к нулю при $x_{0} \rightarrow \infty$. Такой характер зависимости объясняется тем, что с ростом $x_{0}$ снижается эффективность периферийного шунта, которая и определяет величину отпирающего тока управления.

Сравнивая зависимость $J_{G T}\left(x_{0}\right)$ для $\gamma$ - и $\alpha$-тиристоров, легко видеть, что отпирающий ток управления $\alpha$-тиристора равен нулю при $x_{0}=0$ и возрастает с ростом $x_{0}$, в то время как для $\gamma$-тиристора он, напротив, максимален при малых $x_{0}$ и падает, стремясь к нулю при увеличении $x_{0}$.

Второе важное отличие $\gamma$ - и $\alpha$-механизмов переключения, связанное с влиянием неодномерных процессов, заключается в том, что в практически важном диапазоне значений $x_{0}$ отпирающий ток управления $\gamma$-тиристора более чем на порядок превосходит отпирающий ток управления $\alpha$-тиристора.

\section{4. Заключение}

Проанализированы особенности включения в неодномерном приближении $\mathrm{SiC}$-тиристоров, для которых характерен новый, $\alpha$-механизм включения, определяемый токовой зависимостью транспортного коэффициента усиления составного транзистора с блокирующей базой. Показано, что в таких структурах ток управления локализован в узкой области вблизи внешнего края эмиттера. Характерная глубина проникновения тока управления для высоковольтных $\mathrm{SiC}$-тиристоров составляет $x_{0} \sim 400-600$ мкм, что значительно меньше, чем размер современных мощных $\mathrm{SiC}$-тиристоров. Исследовано влияние распределенной утечки на параметры включения такого тиристора. Введение распределенного поверхностного сопротивления приводит к росту включающего прибор тока управления. Однако введение распределенной поверхностной утечки позволяет реализовать ситуацию, когда сигнал тока управления 
проникает на значительное расстояние от внешнего края эмиттера. Полученные зависимости сопоставлены с соответствующими зависимостями для тиристора, включение которого определяется стандартным для $\mathrm{Si}$-тиристоров $\gamma$-механизмом включения, определяемым ростом величины коэффициента инжекции эмиттера, примыкающего к узкой базе, $\gamma_{1}$ с ростом тока. Для таких тиристоров ток включения монотонно растет с уменьшением расстояния от шунта до внешнего края эмиттера, что соответствует росту тока управления с увеличением плотности шунтировки эмиттера.

Работа выполнена при поддержке РФФИ (грант 16-08-01038) и частичной поддержке Wolfspeed, A Cree Company.

\section{Список литературы}

[1] M.E. Levinshtein, S.L. Rumyantsev, T.T. Mnatsakanov, A.K. Agarwal, J.W. Palmour. "SiC thyristors“. In: SiC Materials and Devices, ed. by M. Shur, S. Rumyantsev, M. Levinshtein (World Scientific, Singapore-New JerseyLondon-Hong Kong), 2006) v. 1.

[2] A. Ogunniyi, H. O’Brien, A. Lelis, C. Scozzie, W. Shaheen, A. Agarwal, J. Zhang, R. Callanan, V. Temple. Sol. St. Electron., 54, 1232 (2010).

[3] S.L. Rumyantsev, M.E. Levinshtein, M.S. Shur, T. Saxena, Q.J. Zhang, A.K. Agarwal, J.W. Palmour. Semicond. Sci. Technol., 27, 015012 (2012).

[4] S.L. Rumyantsev, M.E. Levinshtein, M.S. Shur, L. Cheng, A.K. Agarwal, J.W. Palmour. Semicond. Sci. Technol., 28, 125017 (2013).

[5] T.T. Mnatsakanov, S.N. Yurkov, M.E. Levinshtein, A.G. Tandoev, A.K. Agarwal, J.W. Palmour. Sol. St. Electron., 47, 1581 (2003).

[6] M.E. Levinshtein, P.A. Ivanov, T.T. Mnatsakanov, S.N. Yurkov, A.K. Agarwal, J.W. Palmour. Sol. St. Electron., 47, 699 (2003).

[7] Т.Т. Мнацаканов, С.Н. Юрков, А.Г. Тандоев. ФТП, 39, 372 (2005).

[8] M.E. Levinshtein, T.T. Mnatsakanov, A.K. Agarwal, J.W. Palmour. Semicond. Sci. Technol., 26, 055024 (2011).

[9] M.E. Levinshtein, T.T. Mnatsakanov, P.A. Ivanov, J.W. Palmour, S.L. Rumyantsev, R. Singh, S.N. Yurkov. IEEE Trans. Electron. Dev., 48, 1703 (2001).

[10] T.T. Mnatsakanov, S.N. Yurkov, M.E. Levinshtein, L. Cheng, J.W. Palmour. Semicond. Sci. Technol., 29, 055005 (2014).

[11] S.N. Yurkov, T.T. Mnatsakanov, M.E. Levinshtein, L. Cheng, J.W. Palmour. Semicond. Sci. Technol., 29, 125012 (2014).

[12] A.I. Uvarov. In: Physics of $p-n$ junctions and semiconductor devices, ed. by S.M. Ryvkin, Yu.V. Shmartsev (Consultants Bureau-N. Y.-London, 1971) p. 170.

[13] B.J. Baliga. Fundamental of Power Semiconductor Devices (Springer Science, 2008).

[14] M.E. Levinshtein, S.L. Rumyantsev, M.S. Shur, T.T. Mnatsakanov, S.N. Yurkov, Q.J. Zhang, A.K. Agarwal, J.W. Palmour. Semicond. Sci. Technol., 28, 015008 (2013).

[15] В.А. Кузьмин, С.Н. Юрков. РЭ, 25, 1264 (1980).

[16] П.Г. Дерменжи, Ю.А. Евсеев. РЭ, 17, 2365 (1972).

[17] A.V. Gorbatyuk, P.B. Rodin. Sol. St. Electron., 33, 387 (1990).
[18] Н.3. Вагидов., 3.С. Грибников, А.Н. Коршак, В.В. Митин. ФТП, 29, 1958 (1995).

[19] Q. Zhang, A. Agarwal, C. Capell, M. O’Loughlin, A. Burk, J.W. Palmour, V. Temple, A. Ogunniyi, H. O’Brien, C.J. Scozzie. Proc. Int. Conf. on Silicon Carbide and Related Materials ICSCRM2011, September 11-16 2011 (Cleveland, Ohio, USA) p. 409.

[20] N.H. Fletcher Proc. IRE, 43, 551 (1955).

[21] Б. Шкловский, А. Эфрос. Электронные свойства легированных проводников (М., Наука, 1979).

[22] T.T. Mnatsakanov, M.E. Levinshtein, P.A. Ivanov, J.W. Palmour, M. Das, A.K. Agarwal. Semicond. Sci. Technol., 20, 62 (2005).

[23] V.S. Yuferev, M.E. Levinshtein, P.A. Ivanov, Q.J. Zhang, A.K. Agarwal, J.W. Palmour. ФТП, 47, 1068 (2013).

Редактор Г.А. Оганесян

\section{Impact of non-one-dimensional effects on switched-on gate current in $4 \mathrm{H}$-SiC thyristors}

S.N. Yurkov' ${ }^{1}$, T.T. Mnatsakanov' ${ }^{1}$, M.E. Levinshtein ${ }^{2}$, A.G. Tandoev ${ }^{1}$, J.W. Palmour ${ }^{3}$

${ }^{1}$ All-Russian Electrotechnical Institute, 111250 Moscow, Russia

${ }^{2}$ loffe Institute, 194021 St. Petersburg, Russia

${ }^{3}$ Cree Inc., 4600 Silicon Dr.,

Durham NC 27703, USA

Abstract Impact of non-one-dimensional effects, caused by the spread of gate current in the base layer, on switched-on gate current in $4 H-\mathrm{SiC}$ thyristors has been considered. It is shown that new switching mechanism, that is characteristic for $4 H-\mathrm{SiC}$ thyristors, results in the dependence of gate current on parameters of a thyristor, which differs qualitatively from corresponding dependence in traditional silicon thyristors. 